\title{
El descenso de las estrellas. El mundo originario y el origen del mundo en el pensamiento de J. Patočka
}

\author{
Descending from the stars. The originary world and the origin of \\ the world en J. Patočka's thought
}

ÁNGEL ENRIQUE GARRIDO-MATURANO*

\begin{abstract}
Resumen: El artículo analiza desde una perspectiva fenomenológica y hermenéutica el modo originario de donación del mundo y el origen del mundo en el pensamiento de Jan Patočka posterior a 1965. Primero muestra en qué sentido tanto la donación del mundo como horizonte, cuanto los movimientos de la existencia humana, implican un vínculo esencial entre el modo originario de darse del mundo y la noción de infinito, concebido como lo indefinido e inagotable. Finalmente explicita por qué el vínculo del mundo con lo infinito reposa sobre un impulso creativo divino que constituye el fundamento cosmológico de la religiosidad humana.
\end{abstract}

Palabras clave: Patočka, mundo, horizonte, origen, lo divino.

\begin{abstract}
The article analyzes from a phenomenological and hermeneutic standpoint the originary mode of givenness of the world and the origin of the world in Jan Patočka's thought after 1965. First, it shows the sense in which both the givenness of the world as horizon and the movements of human existence imply an essential link between the originary mode of givenness of the world and the notion of infinitude conceived as the indefinite and inexhaustible. Finally, the article explains why the link between the world and infinitude rests on a creative divine impulse that constitutes the cosmological ground of human religiousness.
\end{abstract}

Key-words: Patočka, world, horizon, origin, the divine.

\section{Introducción}

Dirigir la vista hacia la inmensidad celeste, tratando de hurgar allí las huellas inciertas de aquella remota "gran explosión", con la que, presuntamente, comenzó el universo, no le es extraño a la cosmología contemporánea. Sin embargo, la pregunta acerca del porqué del ser-originador de semejante estallido no es planteada por la investigación científica con frecuencia equiparable. Es que la propia formulación de la pregunta por el origen nos

Recibido: 28/06/2018. Aceptado: 12/11/2018.

* CONICET-Instituto de Investigaciones Geohistóricas, Resistencia, Argentina. Investigador Principal. Correo electrónico: hieloypuna@hotmail.com. Línea de investigación: fenomenología posthusserliana, filosofía de la existencia, filosofía de la religión. Publicaciones recientes:"La contracción del horizonte. Contribuciones introductorias a una fenomenología de la pasión”, aceptado para su publicación por: Logos. Anales del seminario de metafísica de la Universidad Complutense, Vol 50 (2017), pp. 129-147. "Un vislumbre de lucidez. Análisis fenomenológico de la noción kierkegaardiana de instante” Signos Filosóficos, México, vol. XIX, No. 38 JulioDiciembre, 2017, pp. 8-33. 
lanza al reino de la paradoja. ¿Cuándo y dónde ocurrió aquello que precede a todo dónde y a todo cuándo? ¿Cómo puede ser algo real lo que funda todo algo y toda realidad? Se trata, en última instancia, de la pregunta por "cómo puede ser pensable el comienzo de la realidad dentro de la realidad (...) y, a una con ello, por la existencia del origen de todo existir" (Schmied, 2007, 61). En el marco de la filosofía fenomenológica me atrevería a decir que uno de los intentos más lúcidos (no, por supuesto, de responder de modo definitivo semejante pregunta, pero sí) de plantear las condiciones trascendentales capaces de afrontar la paradoja de la pregunta por el origen del mundo se la debemos al filósofo checo Jan Patočka. Las consideraciones siguientes se ocuparán del tema de la relación entre la noción de mundo y la de origen en su pensamiento. Lo harán desde dos perspectivas diferentes, pero complementarias. Por un lado, desde el punto de vista del modo originario de donación del mundo. Por otro, desde la consideración del origen último de la donación del mundo. Ellas no pretenden ser una exposición minuciosa de la noción de "mundo natural" en el pensamiento del filósofo ${ }^{1}$, ni mucho menos un estudio genético de la evolución de este concepto a lo largo de su obra². Por el contrario, mi análisis se limita a los textos del último lustro de la década del sesenta ${ }^{3}$, en particular: "Notas sobre la prehistoria de la ciencia del movimiento: el mundo, la tierra, el cielo y el movimiento de la vida humana", que data de 1965; "El mundo natural y la fenomenología", de 1967; y "Meditación sobre «el mundo natural como problema filosófico»", de 1969. Ello se debe a que la investigación persigue dos objetivos que resultan de la interpretación de las obras que despliegan la comprensión del mundo como origen y del origen del mundo en esta época. El primero radica en reconstruir lo que el filósofo entiende por "mundo" durante este período, a los efectos de mostrar cómo al mundo le pertenece un vínculo esencial con lo infinito. En este sentido, parto de la hipótesis según la cual tanto el modo originario de darse del mundo, cuanto el movimiento por el cual el hombre se relaciona con la donación del mundo, testimonian la relación de lo infinito con lo finito, a saber: la posibilitación de las infinitas figuras de lo finito por un horizonte infinito. El segundo, que es una consecuencia del primero, aspira a mostrar cómo este vínculo esencial entre mundo e infinito reposa sobre un impulso creativo de carácter cosmológico que constituye el fundamento último de la religiosidad humana. Aquí parto de la hipótesis de que en el movimiento mismo que hace ser al mundo como tal alienta un "encaje" (Fuge) que bien podríamos llamar "divino".

1 Para ello recomendamos el erudito trabajo de Petricek, M. (1990/1991), «Jan Patocka et l'idée du monde naturel», Les Cahiers de Philosophie. Jan Patocka. Le soin de l'ame, n 11/12, pp. 117-152.

2 En lo que a la evolución del pensamiento fenomenológico de Patočka respecto del tema del mundo hay dos estudios que merecen ser mencionados. Primero el de Srubar, I. (1991), «Zur Entwicklung des phänomenologischen Denkens von Jan Patočka», en: J. Patočka: Die Bewegung der menschlichen Existenz. Phänomenologische Schriften II, Stuttgart: Klett-Cotta, pp. 7-29. Segundo, el que -para mí- es la mejor indagación de la evolución de la idea de mundo en Patočka. Me refiero al estudio de Karfík, F. (2008), «Die Odyssee des endlich gewordenen Absoluten», en: ídem: Unedlichwerden durch die Endlichkeit. Eine Lektúre der Philosophie Jan Patočkas, Würzburg: Könighausen \& Neumann, pp. 32-68.

3 Quienes se interesen por la unidad de mundo y subjetividad trascendental en la producción temprana de Patočka pueden acudir a: Novotný, K. (1999), «Erscheinung des Ganzen. Patočkas phänomenologische Philosophie der dreißiger Jahre bezüglich seines Spätwerks», en: L. Hagedorn y H. Sepp (eds.): Jan Patočka. Texte. Dokumente. Bibliographie, Freiburg/München: Alber, pp. 137-175. 
Desde el punto de vista metódico la investigación se comprende a sí misma como fenomenológico-trascendental y hermenéutica. Es fenomenológica en cuanto respeta el principio metódico "onto-fenomenológico" que rige el conjunto de las investigaciones de Patočka durante este período y que el autor formula en los siguientes términos: "El ente es dado a través del fenómeno, pero, a través del fenómeno, lo que se da es el ente" (Patočka, 1991, 32). De acuerdo con ello, su fenomenología coincide con la husserliana cuando esta última afirma que no podemos acceder a la cosa si no es a través de su aparición; igualmente le concede razón cuando sostiene que la fenomenología no describe el "ser en sî" de los entes, sino su modalidad de donación. Pero insiste en que a través del fenómeno lo que se muestra son en verdad las cosas. No es, entonces, la conciencia, concebida como constitución intencional del ser del objeto, el lugar donde aparece el universo, sino el aparecer del universo el lugar donde puede ocurrir el sujeto y los fenómenos que intenciona. Este principio metódico será fundamental para comprender el sentido de la noción de mundo aquí descripta. Además, se tendrá presente constantemente la función trascendental de principio mencionado, toda vez que el movimiento ontológico por el cual el mundo viene al aparecer es condición de posibilidad de la existencia humana y determina su consumación como relación con lo infinito. Finalmente, el análisis onto-fenomenológico del mundo requiere complementarse con una hermenéutica de la donación, en la medida en que es menester la explicitación de las implicancias del darse del mundo como movimiento onto-fenomenológico para que se ponga de manifiesto su vínculo con lo infinito y su relación con lo divino.

\section{La donación originaria del mundo}

\subsection{El horizonte infinito}

El problema del "mundo natural", es decir, de si hay un modo natural y objetivo de darse el mundo en sí mismo, surge, para Patočka, con el mecanicismo, que se halla marcado por notorios presupuestos metafísicos. Nuestro filósofo observa que lo que existe para la visión mecanicista "es algo construido por el pensamiento" (Patočka, 2004, 14). El instrumento del que éste se vale para edificar su representación de las conexiones objetivas que rigen el mundo es, paradójicamente, una actividad espontánea del espíritu humano: la matemática. De allí que la condición artificiosa de la metafísica mecanicista se observe en la separación entre un mundo matematizado y objetivo de cosas en sí, del que paradójicamente nunca tenemos experiencia directa, y nuestro mundo vital inmediato, que se comportaría como un mero reflejo subjetivo e imperfecto del "verdadero" mundo objetivo. Sin embargo, tanto el mecanicismo cuanto el positivismo y las visiones cientificistas del mundo asumen de manera acrítica dos premisas metafísicas. La primera, la de que las relaciones matemáticas representan la realidad objetiva, sin investigar cómo ellas llegan a ser experimentadas. Así se introduce una estructura matemática no sólo como causa de la vivencia, sino en la propia vivencia. La segunda premisa es aquella por la cual todo lo que es debe verificarse en un plano de certeza objetiva, pero la certeza no es una cuestión de los datos, sino un modo de vivencia del sujeto. Siguiendo la crítica a la que sus maestros Husserl y Heidegger sometieron a esta visión cientificista, Patočka concluye 
que la imagen objetiva y matemática del mundo natural es justamente eso: una imagen o representación subjetiva del mundo; y que la ciencia en su conjunto no es sino "una continuación y extensión más y más sofisticada de la práctica originariamente simple de la objetivación" (Patočka, 1988, 3). Dicha práctica encontraría su origen en las técnicas de medida y comparación de las que se vale la vida cotidiana para poder determinar con precisión un cierto aspecto de las cosas, de modo de poder volver a ellas y utilizarlas de manera idéntica. La ciencia reposa, en última instancia, en la hipótesis, imposible de verificar, de la naturaleza objetiva y calculable de la totalidad del universo. Por lo tanto no se puede afirmar que ella haya alcanzado la verdad absoluta del mundo. Por el contrario, "no es más que un método, un modo de encaminarse en el mundo, y sus resultados no pueden ser proclamados como la realidad misma” (Patočka, 1988, 3).

La ciencia no nos da, pues, el mundo en su realidad absoluta, sino que es una representación subjetiva y artificial de un cierto estado de cosas. Pero lo determinante aquí radica en que ella presupone una situación dada, sobre cuyo trasfondo objetiva las relaciones entre las cosas que se manifiestan desde ese trasfondo. Precisamente ese entorno, en que ya siempre se muestran las cosas susceptibles de ser luego objetivadas por el método científico, es el modo en que originariamente se nos abre el todo del mundo. Éste no es un producto del sujeto, pues no es el sujeto el que decide relacionarse con el ente en una situación ya siempre orientada y abierta a un entorno que se prolonga más y más, sino que es el mundo el que se da a sí mismo como trasfondo en relación con el cual aparece en perspectiva todo lo que aparece y es percibido. El mundo se mueve hacia nosotros y se nos da como trasfondo o campo de aparición en el que lo que aparece ya siempre aparece en la perspectiva y modo en que aparece. Y como no es posible un darse neutro y sin perspectiva de la cosa, el mundo es siempre aquel trasfondo situacional que viene por sí mismo al aparecer no como cosa, sino como confín de la aparición de cada cosa. Éste es precisamente el camino que recorre la onto-fenomenología patočkiana, a saber: acceder al modo primigenio y originario de darse del mundo no desde una actividad subjetiva y artificiosa de matematización, sino desde la percepción en que las cosas son dadas a los sentidos y que constituye la fuente de toda objetivación ulterior. Puesto que cada cosa sensible se da y es percibida en una cierta situación, desde una perspectiva y, por tanto, con una orientación, toda percepción se inscribe ya en una totalidad. En este sentido puede decirse que "el todo previo está presente del mismo modo que las cosas singulares" (Patočka, 1988, 4). Sin embargo, no lo está del mismo modo que aquello que afecta de forma directa a los órganos sensoriales. "Nosotros decimos de modo figurado que la totalidad está ahí como un horizonte" (Patočka, 1988, 4). Cada singularidad nos viene desde esta totalidad o, para ser más exactos, el modo en que se mueve hacia nosotros todo lo que es configura un horizonte de aparición desde el cual cada cosa singular se da al sujeto en un determinado cómo y al cual se retira de nuevo cuando nos apartamos de ella. Esta totalidad previa y presupuesta por el darse de la cosa es el modo en que ya siempre y necesariamente se da el mundo, a saber, como la apertura de un horizonte en el que es posible que se configure el aparecer de todo lo que aparece. Por eso afirma el autor que es necesario caracterizar descriptivamente el horizonte del mundo "como algo originario, que no puede ser compuesto a partir de las impresiones, ni de sus capas representadas ni de la combinación de ambas" (Patočka, 1988, 4). Por eso también afirma su comentarista 
Petricek que "la donación de horizonte es originaria; es una tesis más allá de la cual es imposible ir" (Petricek, 1990/1991, 131). Si se ha llegado a convertir el mundo en el producto de la espontaneidad del sujeto, ello "sólo está «legitimado» por el prejuicio que establece el privilegio de la subjetividad" (Petricek, 1990/1991, 131). En consecuencia, el mundo se da originariamente por sí mismo como horizonte implícito y no es el resultado de ninguna representación subjetiva susceptible de ser matematizada. Él acontece como aquello que "no está perceptivamente presente" (Patočka, 2004, 30), pero que no puede ser puesto entre paréntesis ni reducido en modo alguno, puesto que es aquella "donación no sensible" (Patočka, 2004, 30) desde la cual algo puede explícitamente presentarse. Es la "inmensidad inaparente" (Patočka, 1988, 5) que acompaña y posibilita todo aparecer. Se trata de una totalidad que nunca puede ser traída a la intuición como una omnipresencia actual, pues, si así lo fuera, la presunta totalidad no integraría todo lo que es, sino que se configuraría y recortaría como tal totalidad desde su propio horizonte de mundo. De allí que pueda afirmarse que "el horizonte no es una intención que pueda esperar cumplimiento" (Patočka, 2004, 30), sino que mienta una totalidad integral no actual desde la que emerge y en la que se sumerge lo que llega a ser actual y deja de serlo.

Ahora bien, si las cosas pueden darse orientadas y en una cierta perspectiva, ello se debe, además, a que la percepción de los fenómenos se proyecta desde un centro dado por el cuerpo propio. Todo fenómeno se da en una situación. Pero, para ello, yo tengo que estar corporalmente en la situación. La orientación y la perspectiva se producen a través del cuerpo viviente. Es en función de los movimientos a través de los cuales el cuerpo realiza sus posibilidades vitales que nos dirigimos hacia el mundo y orientamos nuestras percepciones. El movimiento funciona, así, como el vehículo que permite que nos salga al encuentro todo lo que puede aparecer en el mundo. Sin embargo, el hecho de que el movimiento del cuerpo viviente, concebido como la corriente efectuadora de nuestra propia vida, sea aquello en virtud de lo cual nos dirigimos al mundo, no significa, por supuesto, que la orientación sea exclusivamente una orientación hacia el cuerpo. "El centro de referencia de la orientación corporal se encuentra fuera del cuerpo propio" (Patočka, 2004, 33). Toda orientación es orientación hacia afuera, en última instancia, hacia el horizonte del mundo, cuyos referentes máximos son, para Patočka, la Tierra y el cielo.

La Tierra funge como el sustrato inmóvil en que se sostiene la acción orientada. No podemos dirigirnos hacia aquello que emerge en el horizonte si no es sobre el soporte firme que ofrece la Tierra. Nuestras percepciones son percepciones de un cuerpo viviente que se mueve desde la Tierra y que se orienta hacia los objetos que percibe en función del suelo que ella le ofrece. "La tierra es la horizontal natural en relación con la cual tomamos una postura y tenemos una posición" (Patočka, 2004, 34). Pero, además de soporte y primer referente de todas las relaciones, la Tierra aparece también como fuerza y poder. Fuerza, pues sus movimientos, desde las corrientes de agua que la surcan hasta los sismos que la estremecen, dominan los movimientos de todo lo que en ella vive. Y poder, porque reina sobre todos los seres, que sólo logran erguirse sobre su suelo si ella los sostiene y alimenta. La Tierra es, pues, sostén no sólo como suelo, sino también como poder que nutre la vida y puede terminar con ella.

La Tierra no es, con todo, el único referente. Es el suelo bajo nuestros pies y, por ello, el referente de proximidad. Sin embargo, hay otro referente. Éste lo es incluso de la Tierra. 
Se trata de aquel ámbito inconmensurable en el que la Tierra misma se mueve y hacia el que se abren todos los horizontes: el cielo. Él es el referente al que pertenece lo intangible y lejano, pues al cielo, nos elevemos cuanto queramos, nunca se llega: siempre está más allá. Pero su lejanía le concede a lo cercano su "cuándo", pues del cielo proviene la luz y la oscuridad, el día y la noche, y la trayectoria de la Tierra en él marca el curso de los años. El tiempo no es sino el sucederse del movimiento de lo que se da en el cielo. Él le dispensa a todo, además, su "dónde", porque incluso la Tierra se ubica en función de su posición en el cielo. El espacio es la extensión de los cielos. En conclusión, todo encuentro con los entes intramundanos tiene lugar "contra este trasfondo de Tierra y cielo, que desde luego no es siempre ni necesariamente objeto de consideración temática” (Patočka, 2004, 36). Así concebidos, Tierra y cielo no pueden reducirse ni a útiles manipulables ni a cuantificaciones matemáticas, pues, a diferencia de las cosas intramundanas, ellos "tienen la posibilidad eminente de revelar el contexto" (Patočka, 2004, 37). Por esta razón ambos dos pero -a mi modo de ver- eminentemente el cielo, dada su condición de referente de la Tierra misma, "suscitan un vértigo singular en el que el mundo aparece como mundo en su prodigiosa extrañeza, en su prodigio" (Patočka, 2004, 37).

¿Por qué el cielo suscita vértigo y nos indica la extrañeza prodigiosa del mundo? El vértigo es solamente posible ante lo inabarcable, ante aquello que se aleja hacia el "más allá" de toda mirada, hacia lo que está fuera de alcance y se sigue abriendo allende todo límite. En una palabra, el vértigo es siempre vértigo ante lo in-finito. Y lo infinito es, en comparación con todo ente manipulable y cuantificable, lo que resulta extraño y prodigioso. El mundo se da originariamente como horizonte no cerrado, sino siempre abierto a un "más allá" inalcanzable, pues cada horizonte remite a otro y así sucesivamente hasta perderse en la infinitud del cielo. El mundo se da, entonces, originariamente, como horizonte infinito. Concibiendo el mundo como "inmensidad inaparente", como aquel campo de aparición que propiamente no aparece, pero deja aparecer a lo que aparece, Patočka vincula esencialmente el mundo con lo infinito. En efecto, si el mundo es lo que no aparece, es el trasfondo indeterminable de todo aparecer, entonces él no es representable ni de-terminable. No es posible percibir un "fin" o límite del mundo. Si lo fuera, el mundo en totalidad estaría omnipresente de modo actual. Pero justamente de este modo no puede darse el mundo. Por ello mismo, la idea del mundo como horizonte in-determinable, que se derrama más allá de cualquier límite, implica la idea de infinito. Todo lo finito se recorta en este horizonte infinito del universo. No se trata aquí de la infinitud en un mero sentido ideal-matemático, como extensión cuantitativamente infinita. ${ }^{4}$ Tampoco de un ente omni-

4 Entre los manuscritos de trabajo pertenecientes al legado de Patočka, publicados bajo el título Vom Erscheinen als solchem, se halla uno, que data de los años 70, titulado "El mundo como forma de la experiencia y la experiencia del mundo". Allí Patočka afirma que los "conceptos finito-infinito quizás no sean aplicables, especialmente si la infinitud se entiende en un sentido matemático." Sin embargo inmediatamente reconoce que algunas características de este infinito matemático convergen con la experiencia del mundo como horizonte. "Por ejemplo lo infinito no consiste de partes, las precede, no se disuelve en ellas; no tiene sentido hablar de menos-más como en las magnitudes finitas, sus reglas de potencialidad no se dejan reducir a las relaciones cuantitativas de las magnitudes finitas." La diferencia fundamental por la cual esta idea de infinito no sería aplicable al mundo radica en que "el infinito matemático es en todo sentido sólo algo potencial, mientras que indiscutiblemente hay universo, es actual, es el ser mismo" (Patočka, 2000, 109). Precisamente una infinitud actual, cuya potencialidad no puede ser medida por las reglas de lo finito, es la que aquí proponemos con la idea de lo indefinido como 
potente, Ab-soluto y, por ello, distinto de los finitos. Antes bien, se trata de infinitud en el sentido de una potencia indefinida e indefinible, desde la cual pueden emerger inagotables apariciones siempre finitas. Tal cual observa F. Karfík, el mundo "como fundamento de la aparición de todo lo que aparece es, por cierto, un inagotable: un indefinido, que nunca se puede extinguir en los entes definidos" (Karfik, 2008, 63). Como fundamento inagotable del aparecer, como tras-fondo que nunca puede ser abarcado del todo, el horizonte del mundo es lo infinito viniendo hacia lo finito. Su ser no es algo de-finido y, por tanto, finito, sino puro movimiento ontogénico que en su moverse actualiza la posibilidad y modalidad de lo finito, dejándolo, así, aparecer de un cierto modo. Su mejor índice y referente es el cielo. No sólo la magnitud del cielo es inagotable, pues él genera el tiempo y el espacio, y no hay, por ende, tiempo ni espacio que pudiese clausurarlo ${ }^{5}$, sino que, ante todo, las potencialidades que gestan desde él todo aquello que llega a ser son inagotables. Quizás en este sentido de infinito, a saber, como fundamento indefinido e inagotable que hace posible el aparecer de todo lo finito, la comprensión ontogénica del mundo de Patočka, podría restituir, como el propio autor lo sugiere "la antigua physis (...) en tanto que arché que gobierna todo lo singular” (Patočka, 1988, 100). Este vínculo que lo infinito tiene con lo finito se refrenda en los movimientos a través de los cuales la existencia humana se inserta en el movimiento del todo del mundo, pues ellos desembocan en un movimiento hacia lo infinito.

\subsection{Los movimientos de la existencia humana}

Bien podría preguntarse si este universo infinito no es una proyección subjetiva. Bien podría responderse, como lo hace I. Srubar (1991) que "el campo de aparición mismo no es por cierto subjetivo en el sentido de una constitución o construcción, pero lo es en el sentido de que hace visible al yo, en la medida en que le pre-para las posibilidades de su ser" (p. 27). Srubar interpreta con tino a Patočka, pues no es la existencia efectiva del mundo la que depende de que el sujeto lo proyecte, sino que el sujeto es impensable sin el mundo, porque el propio sujeto es uno de los modos en que se individúa el potencial ontogénico que el mundo es. En virtud del carácter del mundo de ser movimiento, es decir, de acontecer como dynamis por la cual se gestan y realizan las posibilidades de ser de todo lo que llega a ser, incluido el propio sujeto, sería falso decir del campo de aparición que él es el resultado de la libertad de la existencia extática que con-forma un mundo. Este mundo circundante es, en todo caso, un fenómeno derivado que ya acontece en y gracias al horizonte del mundo. "Antes bien, se debería decir no que el hombre se arroja hacia el mundo, sino que se recibe desde éste. Es precisamente en este punto donde Patočka anuda su fenomenología asubjetiva [del mundo AGM] a los resultados de su análisis de los movimientos de la existencia humana" (Srubar, 1991, 28). En efecto, no es el mundo el que llega a ser posible por una conciencia sin mundo, sino que la existencia humana concreta sólo llega a realizarse como movimiento si recibe su ser del mundo. El primero de estos movimientos, en que la exis-

potencial inagotable que lleva a la aparición a todo lo que aparece, pero que no se compone de la sumatoria de apariciones.

5 "De allí que Patočka pueda incluso decir: «el mundo no es en el tiempo, sino el tiempo es el mundo»" (Novotný, 1999, 159). 
tencia, como realización dinámica de posibilidades de ser, consiste, testimonia, por antonomasia, esta posibilitación del existir a través del mundo; más precisamente a través de los otros, que son parte de ese mundo y que reciben al existente en él. A este primer movimiento lo denomina el filósofo "enraizamiento".

El sentido ontológico esencial del primer movimiento lo da a entender el propio Patočka cuando escribe que "el contacto con los otros es el componente primordial, el más importante, de este centro del mundo natural cuyo suelo es la Tierra y cuya periferia es el cielo" (Patočka, 2004, 38). El hombre ha sido generado por el movimiento del mundo, por ello él pertenece al mundo natural, pero con la peculiaridad de hacerlo al modo de un centro. Él es un centro en torno del cual se extiende el mundo como horizonte, pues al hombre y -hasta donde sabemos con certeza- sólo a él se le aparece todo lo que viene al aparecer en el horizonte del mundo. Sin embargo, situado sobre la Tierra y bajo el cielo, no podría haber llegado a ser el centro que él es si el mundo, a través de los otros centros que ha generado, no lo recibiese. El primer movimiento a través del cual el sujeto recién nacido se arraiga en el mundo y comienza a realizarse en él sólo es posible, entonces, sobre la aceptación de ese mismo mundo a través de los otros que cuidan al nuevo ser y ponen a su disposición los frutos de la Tierra. Estos otros son, por excelencia, los padres. No es cuestión aquí de analizar en detalle los tres movimientos y sus diversas implicancias fenomenológicas. Desde el punto de vista ontológico lo determinante de este primer movimiento radica en mostrar que el mundo no es una representación del hombre, sino que el hombre es un fruto del mundo y, como tal, una de las maneras y perspectivas finitas (centros) a través de las cuales el horizonte infinito del mundo hace posible el aparecer de lo que aparece. De acuerdo con ello el mundo no presupone al hombre, sino el hombre al mundo. El sujeto y su libertad no tienen origen en sí mismos. "El yo sólo es fuerza y libertad de responder al llamado del mundo" (Tardivel, 2007, 444). Un mundo que me llama a ser recibiéndome y dándome mis posibilidades de ser a través de los otros. Este primer movimiento puede sintetizarse, entonces, diciendo que en él soy "por o gracias al otro" y que su dimensión temporal por antonomasia es el "pasado", en cuanto aquello que me acepta es lo ya dado, la facticidad no sólo limitante, sino posibilitante del mundo mismo. Finalmente. el lazo que me liga aquí a ese mundo es el del "placer", el gozo de ser aceptado y poder disfrutar de los nutrientes de la Tierra.

Pero luego del enraizamiento la existencia debe prolongarse en el mundo. El movimiento de prolongación o reproducción es el segundo de los tres que realizan la existencia humana. Aquí se trata de apropiarse y poseer las cosas presentes; de utilizar lo que viene al aparecer en el horizonte del mundo de acuerdo con las necesidades de la vida. El movimiento de prolongación se desinteresa del mundo como horizonte infinito y sólo se preocupa por las cosas en la utilidad que éstas tienen ahora para la existencia. El mundo que se ofrece al movimiento de prolongación de sí resulta, entonces, un plexo de útiles compartidos, pues todos deben competir por los recursos que el mundo ofrece para poder continuar con la tarea de vivir. Este segundo movimiento se realiza por excelencia en el orbe del trabajo. En él impera la organización y la jerarquía, que resulta de la lucha por el dominio de los recursos. Cada uno de nosotros se convierte en un centro autónomo de posibilidades, pero el impulso hacia las cosas, que permiten la realización de esas posibilidades, hace que "nos escapemos de nosotros mismos, que no tengamos conciencia de nosotros mismos" (Patočka, 
1988, 115), es decir, que nos consideremos una cosa más, otro engranaje en el sistema productivo. Igualmente, el otro aparece cosificado, "en la medida en que es integrado en un sistema de preocupaciones destinadas a satisfacer necesidades" (Patočka, 1988, 116). El hombre queda reducido a un rol social. Lo determinante desde el punto de vista ontológico de este segundo movimiento, es que en él se oculta la relación del hombre con el mundo como horizonte infinito, para dejar surgir el mundo como una totalidad limitada de útiles y recursos. Pero como la ocultación es uno de los modos de la mostración, él sólo es posible sobre la base del originario darse del mundo como horizonte infinito. Sintéticamente podría caracterizárselo diciendo que el éxtasis temporal que en el domina es el presente, pues depende de la realización actual de mi existencia en relación con los útiles dados. El otro me sale al encuentro como aquel "contra" y no "por" el que soy, pues compite conmigo por la realización de nuestras posibilidades respectivas. Y el vínculo que me liga al mundo es el de la lucha y el trabajo.

En el movimiento de prolongación de sí hay siempre algo indomeñable: la muerte. Ella nos enfrenta a una suerte de ausencia que la organización de las técnicas de producción no puede llenar. Una ausencia que se va intensificando a medida que tomamos conciencia de nuestra mortalidad, hasta que ella termina rodeándonos. Inmerso en ella, el existente puede desesperar, engañarse a sí mismo y negarla. Puede absorberse en el incremento de la efectividad de las técnicas de prolongación. Pero puede también moverse hacia lo que esa ausencia le señala y buscar lo que el segundo movimiento no puede darle: el sentido del conjunto de su vida, el sentido de su paso por el mundo. Cuando ello ocurre el existente pasa al tercer movimiento: el movimiento de apertura a y penetración en la verdad. Se trata de un movimiento de dedicación de sí que conlleva al redescubrimiento del mundo como horizonte infinito. El movimiento de apertura es el más importante de los tres. ¿Por qué? En los primeros movimientos la vida me encadena a mí mismo: al ejercicio y preservación de mis funciones vitales, mientras que en el tercero, en el que asumo mi finitud y busco un sentido para ella, puedo transformar mi ligazón a mi propia vida y, a la par, modificar mi relación con el conjunto del universo. En efecto, afrontar la finitud significa experimentar que poner el fin en mí mismo es poner el fin en lo que está destinado a la nada, y que, consecuentemente, haciéndolo, reduzco a nada el sentido de mi existencia. Allí emerge la ausencia de la que hablábamos; y allí también se abre la posibilidad de la búsqueda de un sentido para mi vida finita que no quede prisionero de mi propia finitud. De aquí en adelante se abre también la posibilidad de ser para más que para mí mismo. La asunción de la finitud adquiere, entonces, la significación de la dedicación y entrega al ser de todo lo que es. Escribe el filósofo:

Mi ser ente no está ya más definido como un ser para mí, sino como un ente en la dedicación, un ente que se abre al ser, que vive para que las cosas sean, para que las cosas - y también yo mismo y los otros- se muestren en lo que ellas son. (Patocka, $1988,122)$

Consumar la propia vida como un movimiento de dedicación quiere decir concretamente vivir en la devoción; tener el centro de sí fuera de sí. Pero no por una simple concordancia de intereses, sino por un vuelco del interés, que ya no se centra en mí, sino que se dirige al 
universo entero: a poner mi vida al servicio del aparecer de siempre nuevas y potencialmente infinitas modalidades de ser. El ser en devoción es el ente que se dedica al ser de todo y que por ello no pierde, sino que, por el contrario, descubre el mundo como horizonte infinito de aparición de inagotables posibilidades de ser. Éste es precisamente el sentido ontológico determinante del tercer movimiento, en el que se pasa del "contra el otro" al "para el otro", del encadenamiento al presente a la posibilitación del advenir, y de la lucha por sí al sacrificio por otro, a saber: el descubrimiento del mundo como horizonte infinito y el concomitante ser-para la infinitud del mundo. Si el movimiento infinito del mundo gesta un ser que, además de ser, es fenomenológico, en cuanto centro finito al que se le aparece lo que aparece en el mundo, ahora a este centro se le aparece el mundo mismo como horizonte infinito de potencialidades de ser y, a una con ello, convierte su existencia finita en movimiento al servicio de esa infinitud. El llamado del mundo a hacer ser mi propio ser, que ocurría en el primer movimiento, desemboca en el tercero en la puesta de mi libertad al servicio de ese mismo mundo. El mundo que fue el sentido -aquello por lo cual- la existencia es posible, deviene ahora el sentido -aquello para lo cual- ella es. El "por" se transforma en "para", completando, así, la gesta del sentido e imbricando el movimiento de la existencia en el del mundo. En términos de A. Matousek:

El sentido determina a la vez la fenomenalidad de lo fenomenalizado, y la manera en que nosotros estamos abiertos a él. Estas dos maneras de ser del sentido son inseparables y están mutuamente ligadas, y su ligazón es, en la filosofía de Patočka, concebida y expresada por medio del concepto del movimiento de la vida. (Matousek, 1990/1991, 153)

En el movimiento de la vida se aúnan el sentido del mundo como venir a la fenomenalidad de lo fenomenalizado y el de nuestra existencia, que encuentra su sentido en ser para este advenimiento. Ciertamente ello se cumple en el tercer movimiento, pero Matousek tiene razón al hablar del movimiento de la vida en general, pues él no sería posible sin los dos precedentes.

La vida en la devoción resulta también una suerte de vida infinita. No, obviamente, porque la muerte individual sea allí suprimida, sino porque la vida pasa a tener su centro fuera de sí. Como vida dedicada al servicio de lo que supera al individuo finito, llega a ser "una vida cosmocéntrica y luminocéntrica" (Patočka, 1988, 122). De tal suerte pasa también a formar parte de la infinitud del cosmos, que se da originariamente como inagotable fuente de luz, de fenomenalidad y de sentido. Una lectura sesgada podría tachar de "mística" esta comprensión del modo originario de darse del mundo, que Patočka nos describe, pero, entonces, podríamos responder, con él, "que ella no tiene nada de «místico», a menos que queramos calificar con ese término el mundo mismo, el ser y hasta el hombre, este ser «del» mundo, que vive en una relación esencial al mundo y al ser" (Patočka, 1988, 124).

\section{El origen del mundo}

El mundo se da de modo originario, tal cual vimos, como horizonte infinito a partir del cual se gesta y llega a ser todo lo que es. En este sentido, es un movimiento ontogénico. Pero 
él gesta también aquel centro que no sólo es, sino que es "fenomenológico", en cuanto a él le aparece todo lo que, desde el horizonte infinito del mundo, llega a aparecer. Así concebido, es un movimiento, además de ontogénico, fenomenalizante. 6 Ahora bien, ¿por qué lo es? ¿Qué es lo que hace que el mundo acontezca como mundo, esto es, como movimiento ontogénico y fenomenalizante? $\mathrm{O}$, dicho de otro modo, ¿cuál es el origen del mundo? Desde la perspectiva de Patočka esta pregunta podría formularse también en estos términos: ¿cuál es el origen del sentido? En efecto, el mundo es lo que a todo da ser y el sentido lo es siempre del ser. El mundo no "tiene" un sentido fuera de él, porque fuera de él no hay nada, sino que con él comienza, luce y concluye el sentido. Comienza, porque de él no surge el caos ni la vacuidad insignificante, sino la vastedad de los entes y de sus estructuras y relaciones. Luce, porque él gesta aquellos centros a los que se les aparece, de modos y perspectivas diversas, el sentido de los entes. Concluye, porque el sentido último del ente ante el cual luce el sentido del mundo es "ser-para-el-ser-del mundo" todo.

A mi modo de ver los avances más decisivos de Patočka en lo que a la pregunta por el origen del mundo respecta, se desarrollan en un ensayo breve, pero brillante, de 1972: Universo y mundo del hombre. Observaciones a un planteamiento cosmológico contemporáneo. Allí se parte de la reinterpretación, desde una perspectiva no idealista, de la que el pensador checo califica de "genial idea kantiana" (Patočka, 2004, 85) de la prioridad de la totalidad del espacio respecto de las distintas "limitaciones", es decir, entidades, sistemas y estructuras, que en él tienen lugar. Sobre este espacio no recae originalmente la más mínima significación cosmológica, puesto que es considerado, en el sistema crítico, como una de las formas de la síntesis subjetiva de la experiencia. Sin embargo, en concordancia con aquel principio metodológico según el cual el sujeto presupone el mundo, y no a la inversa, Patočka dota de un tenor ontológico a aquel supuesto "espacio" anterior a las limitaciones. Precisamente en tanto ese "espacio" es anterior a las limitaciones no puede tratarse, en su caso, de un puro espacio vacío. Su prioridad mienta, por tanto, no la anterioridad de un "todo-nada", sino "de aquel "todo-uno" que no puede resultar de la composición de lo múltiple que hay en él. En términos del propio filósofo: "Se supone que la prioridad consiste en que es posible una inabarcable e infinita multiplicidad de limitaciones contingentes en relación con las cuales el todo uno ostenta el rango de presupuesto necesario" (Patočka, 2004, 86). ¿Por qué necesario? Por el mismo hecho de que las distintas limitaciones son incomprensibles como tales si no hay un todo respecto del cual ellas se limitan. A este "todo-uno", que, en tanto anterior y prioritario, es aquello a partir de lo cual se generan las infinitas limitaciones, lo reinterpreta Patočka, apelando a un término dilecto de Heidegger, ya no como espacio vacío, sino como "encaje" ("Fuge"). El todo previo puede ser un espacio abstracto y vacío allí donde no se considera que este todo precedente está ahíto de la posibilidad o impulso creativo de las limitaciones, que, de hecho, han venido al aparecer, y que, en tanto constituyen estructuras interrelacionadas, "encajan" las unas con las otras en un cosmos. El encaje es precisamente aquello que "da a todo lo singular que existe fácticamente, que tiene lugar como «limitación» (...), el lugar

6 En efecto, el movimiento de fenomenalización, aunque se cumple en el hombre, pertenece al mundo, porque aquella estructura fundamental del hombre de estar abierto al ser de las cosas no es obra, sino condición suya. "No es que el hombre sea una condición de posibilidad de tales estructuras, sino que ellas son condición de posibilidad del hombre" (Patočka, 2005, 272). 
y el momento de su existencia, esto es, su aparecer determinado como formación en el interior de otra formación" (Patočka, 2004, 86-87). Sobre la base del encaje, que no es él mismo una limitación que pueda manifestarse, pueden aparecer y articularse espaciotemporalmente el conjunto de las limitaciones que efectivamente aparecen. Dicho brevemente, sobre la base de esta potencialidad creativa de todo puede darse el universo como cosmos. El "encaje" resulta, así, "el oscuro, por indeterminado, fundamento del mundo, determinante de todo y en todo imperante" (Patočka, 2004, 87). Ciertamente este encaje omnímodo no está dado en la percepción sensible, pero puede ser legítimamente supuesto por una fenomenología asubjetiva de la percepción, toda vez que las infinitas limitaciones, como limitaciones, es decir, en tanto aspectos o escorzos de un todo que nunca es asible en totalidad, testimonian ese todo. Dicho con mayor exactitud: testimonian un todo no aparente como im-pulso o potencialidad creativa de esas mismas e inagotables limitaciones que se articulan en un cosmos. Ellas remiten, pues, en última instancia, a aquel conatus o a aquella creatividad que posibilita que haya cosmos y que sus rostros se renueven cada día.

Así concebido, este "encaje" no es ni lo que aparece en el mundo ni el mundo en tanto movimiento que lleva al aparecer. Patočka lo dice explícitamente: "El ajuste del mundo no es ningún ente, ni una cosa ni un movimiento, tampoco un proceso o un acontecer (...)." (Patočka, 2004, 88). No es, entonces, el universo, sino "lo que impera propiamente en el universo uno, inabarcable y dominante sobre todo lo singular" (Patočka, 2004, 88). Antes que al universo, el encaje del que habla el filósofo checo mienta el origen del universo como uni-verso: el origen mismo del cosmos como tal. No es el mundo, sino el fundamento de que haya mundo y de que ese mundo sea un cosmos. Sin aparecer, él es la raíz posibilitante de todos los apareceres y del mundo mismo como movimiento o acontecimiento que lleva al aparecer. Si un adjetivo lo describe, ése es el de infinito, porque no lo abarca ninguna limitación -ni siquiera el propio universo-, sino que es aquello que hace posible el universo como cosmos; y en el cosmos las inagotables e infinitas limitaciones que a él remiten. En una palabra, este encaje, este impulso, este conatus o creatividad que lleva a todo lo que es a ser un uni-verso o cosmos y que hace posible aquellos vivientes a los cuales el cosmos se les manifiesta como tal, es el origen mismo del mundo. A este origen bien lo podemos llamar "lo divino". 7

Lo divino es un término riesgoso "que puede hacer el juego a aquellos que nos reprochan no hacer filosofía, sino teología disfrazada, un reemplazo de la religión” (Patočka,

7 En este sentido es sugestivo, como señala F. Karfík, que Patočka, para referirse a su concepción del mundo como "potencia de todo (dynamis pantôn )" se valga de la expresión "non aliud", que originalmente Nicolás de Cusa usó para uno "de los enigmáticos nombres de Dios como el misterioso origen de todo" (Karfik, 2008, 62). Mi interpretación difiere de la de Karfik en el hecho de que, centrándome en la concepción de "encaje", que aquí hemos interpretado como impulso o creatividad originaria, y en la citada declaración de Patočka en el texto del 72 acerca de que este fundamento último que impera en todo "no es el universo", pensamos que lo divino no puede identificarse con el mundo como lo afirma Karfik (2008): "En Patočka es el mundo como lo non aliud patentemente también un fundamento posibilitador" (p. 63). Antes bien, soy de la idea de que lo divino no es el mundo, sino el origen del mundo, es decir, lo que hace que el mundo acontezca como mundo. Ese origen, si bien sólo puede acontecer en el mundo, no puede, a mi modo de ver, identificarse con el mundo como sumatoria de las parcialidades o limitaciones que hay en él, ni tampoco con el movimiento por el cual el horizonte del mundo trae lo que aparece al aparecer, sino con aquel Misterio que hace que el mundo mismo se mueva. 
1990, 313). Pero no lo es tanto si nos percatamos de que con semejante término Patočka no se refiere a ninguna revelación positiva determinada, ni tampoco a una divinización mística del mundo, sino "simplemente a aquello que domina todo, que está por debajo de todo" (Patočka, 1990, 313) y que traspasa el universo entero como impulso creativo a realizar su infinita riqueza. En tal sentido estamos en pleno derecho de utilizar filosóficamente el término, sin por ello determinarlo ni teológica (en el sentido confesional) ni onto-teológicamente (como si fuese un ente definido al estilo de la causa sui). Lo divino, así comprendido, no es, reitero, el universo -no hay aquí ningún panteísmo-. sino, como el propio Patočka lo señala, "el Misterio" (Patočka, 1990, 238) de que haya universo. Aquel Misterio que, sin dejar de serlo, se deja sentir en la infinita diversidad del cosmos y en el asombroso hecho de que haya un ser capaz de con-moverse ante ella. Es el Misterio que se tras-luce en el horizonte inconmensurable del cielo y en la maravilla de que sus estrellas desciendan hasta volverse tierra y polvo de los que nace un ser que, estremecido, mirará las estrellas.

Así entendida, la cosmología de Patočka, "no es ninguna metafísica que aspire a descubrir un «mundo verdadero» tras los fenómenos, sino el intento de hacer transparentes los propios fenómenos en relación con la totalidad una que se hace presente en ellos mismos" (Patočka, 2004, 92). Así entendida, ella es también el intento de dar, desde la fenomenología, un fundamento cosmológico no sólo a las religiones, sino, mucho antes que ello, a la religiosidad humana.

\section{Referencias bibliográficas}

KARFÍK, Filip (2008): Unedlichwerden durch die Endlichkeit. Eine Lektüre der Philosophie Jan Patočkas, Könighausen \& Neumann, Würzburg,

MATOUSEK, Alexander (1990/1991): "Le «sens naturel» est-il un invariant du monde naturel?", Les Cahiers de Philosophie. Jan Patočka. Le soin de l'ame, n 11/12, pp. 153-162.

NOVOTNÝ, Karel (1999): "Erscheinung des Ganzen. Patočkas phänomenologische Philosophie der dreißiger Jahre bezüglich seines Spätwerks", en: L. Hagedorn y H. Sepp (eds.): Jan Patočka. Texte. Dokumente. Bibliographie, Alber, Freiburg/München, pp. 137-175.

PATOČKA, Jan (1988): Le monde naturel et le mouvement de l'existence humaine, Dordrecht, Kluwer Academic Publishers.

PATOČKA, Jan (1990) : Liberté et sacrifice. Écrits Politiques, Editions Jérôme Millon, Grenoble.

PATOČKA, Jan (1991): Platón y Europa, Península, Barcelona.

PATOČKA, Jan (2000): Vom Erscheinen als solchem. Texte aus dem Nachlaß, Alber, Freiburg/ München.

PATOČKA, Jan (2004): El movimiento de la existencia humana, Ediciones Encuentro, Madrid, 2004.

PATOČKA, Jan (2005): Introducción a la fenomenología, Herder, Barcelona.

PETRICEK, Miroslav (1990/1991): “Jan Patocka et l'idée du monde naturel”, Les Cahiers de Philosophie. Jan Patocka. Le soin de l'ame, n 11/12, pp. 117-152. 
SCHMIED, Alfred (2007): "Der Himmel als Präsenz des Ursprungs. Intentionen antiker Physiko-Theologie", en: E. Angehrn (ed.): Anfang und Ursprung. Die Frage nach dem Ersten in Philosophie und Kulturwissenschaft, Walter de Gruyter, Berlin/ New York, pp. 61-83.

SRUBAR, Ilja (1991): "Zur Entwicklung des phänomenologischen Denkens von Jan Patočka", en: J. Patočka: Die Bewegung der menschlichen Existenz. Phänomenologische Schriften II, Klett-Cotta, Stuttgart, pp. 7-29.

TARDIVEL, Emilie (2007): "La subjectivité dissidente: Étude sur Patočka", Studia Phaenomenologica, vol. VII, pp. 435-463. 